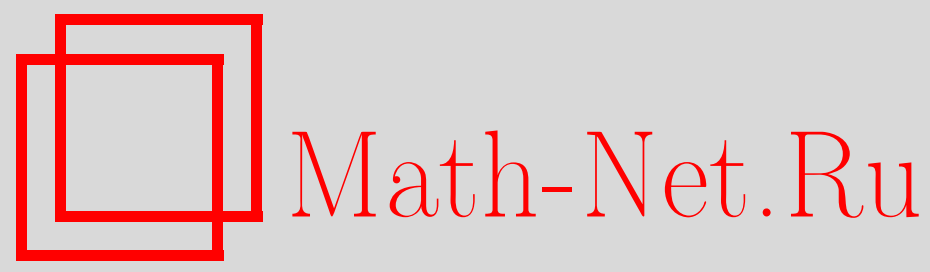

В. А. Зорич, В. М. Кесельман, Конформный тип и изопериметрическая размерность риманова многообразия, Матем. заметки, 1998, том 63, выпуск 3, 379-385

DOI: https://doi.org/10.4213/mzm1292

Использование Общероссийского математического портала Math-Net.Ru подразумевает, что вы прочитали и согласны с пользовательским соглашением http://www . mathnet.ru/rus/agreement

Параметры загрузки:

IP: 54.198 .64 .247

26 апреля 2023 г., 15:49:21

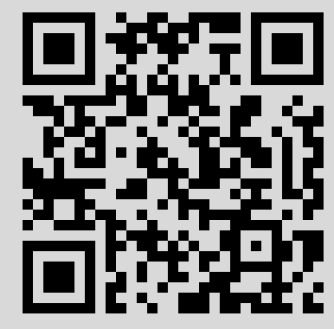




\title{
КОНФОРМНЫЙ ТИП И ИЗОПЕРИМЕТРИЧЕСКАЯ РАЗМЕРНОСТЬ РИМАНОВА МНОГООБРАЗИЯ
}

\author{
В. А. Зорич, В. М. Кесельман
}

Вводится понятие конформной изопериметрической размерности и устанавливается ее связь с конформным типом риманова многообразия.

Библиография: 6 названий.

1. Введение. Классическое для геометрии изопериметрическое неравенство привело к таким понятиям как изопериметрическая функция [1], изопериметрическая размерность [2], изопериметрический профиль [3] и т.п. на произвольном римановом многообразии. Альфорс заметил определенную конформную инвариантность изопериметрических соотношений.

Развивая эти наблюдения, результаты указанных работ и нашей работы [4], мы вводим понятие конформной изопериметрической размерности и устанавливаем ее связь с конформньм типом риманова многообразия.

2. Конформный тип риманова многообразия. Всюду далее мы рассматриваем $n$-мерное некомпактное риманово многообразие, которое обозначаем через $M$ или $(M, g)$, где $g$ - риманова метрика на $M$. Многообразие $M$ назьваем конформно-параболическим или конформно-гиперболическим, если конформная емкость его идеальной гранишы (абсолюта) равна 0 или положительна соответственно. За более развернутыми формулировками приводимых здесь и далее понятий (как правило, выделенных курсивом) мы отсылаем читателя к работе [4] и к цитируемой там литературе.

В этой заметке для нас исходным будет следуюший геометрический критерий конформного типа многообразия (см. [4], [5]), берущий начало при $n=2$ от критерия Альфорса [6] конформной параболичности римановой поверхности.

Теорема 1. Риманово многообразие М размерности $п$ конформноо-параболично тогда и только тогда, когда существует полная, конформная исходной, метрика g на $M$, для которой расходится интеграл

$$
\int^{+\infty} S^{1 /(1-n)}(r) d r
$$

Работа выполнена при частичной поддержке Российского фонда фундаментальных исследований, гранты № 96-01-01218 и № 96-01-00901. 
где $S(r)$ есть g-мера (площадь) геодезической $g$-сферы радиуса $r$.

Напомним, что метрика $\widetilde{g}$ называется конформно-әквивалентной или, просто, конформной метрике $g$, если $\widetilde{g}=\lambda^{2} g$ для некоторой положительной функции $\lambda$ на $M$. Далее при переходе от метрики $g$ к метрике $\widetilde{g}$ одноименные геометрические объекты будут наделяться тильдой.

3. Изопериметрическая размерность риманова многообразия. Справедлива следующая, восходящая к Альфорсу [1] и обобщенная Громовым [2],

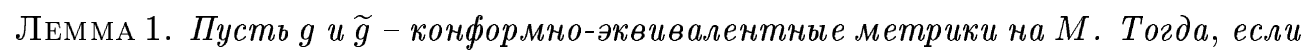
$\widetilde{P}$ - произвольная изопериметрическая функиия в метрике $\widetilde{g}$, то

$$
\int_{\widetilde{V}_{0}}^{\tilde{V}_{1}} \widetilde{P}^{n /(1-n)} \geqslant \int_{r_{0}}^{r_{1}} S^{1 /(1-n)}
$$

əде $\widetilde{V_{0}}, \widetilde{V_{1}}$ суть $\widetilde{g}$-объемы геодезических g-шаров радиусов $r_{0}, r_{1}$ соответственно.

Напомним, что функция $P: \mathbb{R}_{+} \rightarrow \mathbb{R}_{+}$является изопериметрической функиией в некоторой метрике $g$ на $M$, если для всех допустимых областей $D \subset M$ (т.е. имеющих конечные $n$ и $n-1 g$-меры множеств $D$ и $\partial D$, обозначаемые далее через $V(D)$ и $S(\partial D)$ соответственно) выполняется изопериметрическое неравенство

$$
P(V(D)) \leqslant S(\partial D)
$$

Изопериметрической размерностью многообразия $M$ в метрике $g$ называется точная верхняя грань всех таких чисел $p \geqslant 0$, для каждого из которых неравенство вида

$$
V^{p-1}(D) \leqslant c \cdot S^{p}(\partial D)
$$

(где $c$ - некоторая, возможно зависящая от $p$ постоянная) выполняется одновременно для всех допустимых областей $D \subset M$ таких, что $V(D) \geqslant \delta>0$. Последнее условие обеспечивает вьполнимость неравенства (4) при $p=0$, так что изопериметрическая размерность корректно определена в любой метрике.

Непосредственным следствием леммы 1 является (см. [2])

УТВЕРЖДЕНИЕ 1. Если для полного риманова многообразия $(M, g)$ интеграл (1) расходится, то изопериметрическая размерность многообразия в любой конформной $g$ метрике не больше размерности самого многообразия.

Из приведенного утверждения 1 с учетом теоремы 1 сразу получаем

СЛЕДСТВИЕ 1. Если многообразие конформно-параболическое, то в любой метрике, конформной исходной, его изопериметрическая размерность не больше его размерности.

Лемма 1 и последние утверждения показывают, что есть определенная степень конформной инвариантности изопериметрических соотношений. Ниже мы рассмотрим этот вопрос более детально и выявим точньй смысл сказанного. 
Отметим, что хотя в силу следствия 1 изопериметрическая размерность меняется ограниченно при конформных заменах метрик (во всяком случае, на конформно-параболических многообразиях), тем не менее изопериметрическая размерность сама по себе, конечно, не является конформным инвариантом.

Действительно, например, для стандартного бесконечного кругового цилиндра изопериметрическая размерность равна единище, а для стандартной евклидовой плоскости с выколотой точкой она равна 2 , в то время как эти параболические многообразия конформно-эквивалентны.

\section{4. Изопериметрическая функция и изопериметрическая размерность ис-} черпания. Мы собираемся исследовать связь между изопериметрическими соотношениями на римановом многообразии и его конформньпм типом. Поскольку конформньй тип риманова многообразия определяется только структурой многообразия на бесконечности (массивностью абсолюта), естественно рассматривать изопериметрические соотношения лишь применительно к семейству областей, исчерпываюших наше многообразие, например, к семейству геодезических шаров, образуюших геодезическое исчерпание многообразия. Рассматривая изопериметрические соотношения на таком семействе областей, мы естественно приходим к понятию изопериметрической функции и изопериметрической размерности, связанньми с данным исчерпанием.

Уточним, что под исчерпанием многообразия $M$ будем понимать произвольное семейство $E=\left\{D(t) \mid t \in \mathbb{R}_{+}\right\}$предкомпактных областей $D(t) \subset M$ с регулярной границей $\partial D(t)$ такое, что

$$
t_{1}<t_{2} \Longrightarrow \overline{D\left(t_{1}\right)} \subset D\left(t_{2}\right), \quad \bigcup_{t} D(t)=M .
$$

$\Phi$ ункцию $P: \mathbb{R}_{+} \rightarrow \mathbb{R}_{+}$назовем изопериметрической функиией исчерпания $E=\{D(t) \mid$ $\left.t \in \mathbb{R}_{+}\right\}$в некоторой метрике $g$ на $M$, если неравенство (3) выполняется для областей $D=D(t)$ при всех достаточно больших значениях $t$.

Изопериметрической размерностью исчерпания $E=\left\{D(t) \mid t \in \mathbb{R}_{+}\right\}$в метрике $g$ назовем точную верхнюю грань всех чисел $p \geqslant 0$, для каждого из которых вьполняется неравенство (4), когда $D$ пробегает области $D(t)$ при всех достаточно больших значениях $t$.

В работе [4] было показано, что лемма 1 распространяется и на изопериметрические функции исчерпания. А именно, неравенство (2) остается в силе, даже если $\widetilde{P}$ не универсальная (т.е. действующая для любых допустимых областей) изопериметрическая функция в метрике $\widetilde{g}$, а является таковой только для $g$-шаров $B(r), r \in\left[r_{0}, r_{1}\right]$. Поэтому с помощью теоремы 1 получаем следующее

УТВЕРЖДЕНИЕ 2. На конформно-параболическом многообразии $M$ размерности $n$ существует полная, конформная исходной, метрика (удовлетворяющая условию (1) теоремы 1), в которой геодезическое исчерпание Е обладает следующим свойством:

если функиия $\widetilde{P}$ является изопериметрической функиией исчерпания $E$ в какой-либо метрике $\widetilde{g}$, конформной исходной, то расходится интеграл

$$
\int^{\tilde{V}} \widetilde{P}^{n /(1-n)},
$$


где $\widetilde{V}-$ облем многообразия $M$ в метрике $\widetilde{g}$.

Что касается изопериметрической размерности исчерпания, то хотя очевидно, что изопериметрическая размерность многообразия не больше изопериметрической размерности любого его исчерпания (в одной и той же метрике), тем не менее утверждение следствия 1 можно распространить и на изопериметрическую размерность исчерпания.

СлЕДСТВИЕ 2. Если многообразие конформно-параболическое, то существует геодезическое исчерпание (в некоторой полной конформной исходной метрике, удовлетворяющей условию (1) теоремы 1), изопериметрическая размерность которого в любой метрике, конформной исходной, не больше размерности самого многообразия.

Действительно, пусть $E=\{B(r)\}-$ указанное в утверждении 2 исчерпание. Фиксируем произвольную, конформную исходной, метрику $\widetilde{g}$ и обозначим через $d$ изопериметрическую размерность исчерпания $E$ в ней. Тогда для любого положительного числа $p<d$ на исчерпании $E$ вьполняется неравенство вида (4). Значит, функция $\widetilde{P}(x)=c \cdot x^{(p-1) / p}$ является изопериметрической функцией исчерпания $E$ в метрике $\widetilde{g}$. Следовательно,

$$
+\infty=\int^{\tilde{V}} \widetilde{P}^{n /(1-n)}=c \cdot \int^{\tilde{V}} x^{(p-1) / p \cdot n /(1-n)} d x .
$$

Отсюда $\widetilde{V}=+\infty$ и $p \leqslant n$. Ввиду произвольности $p$ получаем $d \leqslant n$, что и доказьвает следствие 2 .

Изопериметрическая размерность исчерпания, так же, как сама изопериметрическая размерность, не является конформным инвариантом риманова многообразия, что видно уже из примера, приведенного в конще п. 3.

ЗАмЕчАниЕ 1. Мы докажем ниже, что утверждение следствия 2 , на самом деле, обратимо (см. следствие 3). Отметим, однако, что даже если изопериметрическая размерность геодезического исчерпания в какой-либо полной метрике, конформной исходной, не больше размерности многообразия, то это еще не означает, что многообразие конформно-параболично. Например, можно построить полное конформно-гиперболическое многообразие $(M, g)$, для которого $S(r)=r^{n-1} \ln ^{n} r$. Нетрудно видеть, что изопериметрическая размерность его геодезического исчерпания в метрике $g$ равна размерности многообразия.

Использование изопериметрической функции исчерпания мотивируется еще и тем, что универсальной изопериметрической функции на всем многообразии может, вообще говоря, не существовать (кроме тождественно нулевой), в то время как для любого исчерпания $E=\left\{D(t) \mid t \in \mathbb{R}_{+}\right\}$в заданной метрике $g$ существует бесконечно много ненулевых изопериметрических функций. Более того, имеется максимальная изопериметрическая функиия для $E$ : таковой является, очевидно, функция $P_{\max }(x):=S(\partial D(t))$, где $x=V(D(t))$.

Отметим, что понятие максимальной изопериметрической функции исчерпания аналогично понятию изопериметрического профиля многообразия [3] в той же мере, в какой понятия изопериметрической функции исчерпания и изопериметрической размерности исчерпания аналогичны соответствующим понятиям изопериметрической функции и изопериметрической размерности многообразия. 
В работе [4] нами получено следующее утверждение относительно максимальной изопериметрической функции геодезического исчерпания.

Лемма 2. Пусть многообразие $(M, g)$ полное. Функиия $\widetilde{P}$ является максимальной изопериметрической функиией исчерпания, геодезического в некоторой сферически конформной $g$ метрике $\widetilde{g}$, тогда и только тогда, когда интеграль (1) $и$ (5) сходятся или расходятся одновременно.

Напомним, что метрика $\widetilde{g}$ называется сферически конформной метрике $g$, если $\widetilde{g}=$ $\lambda^{2}(r) g$, где $r$ - функция $g$-геодезического расстояния. Ясно, что для сферически конформных $g$ метрик и только для них геодезические исчерпания совпадают.

5. Конформная изопериметрическая размерность исчерпания. Мы будем рассматривать далее только конформно-геодезические исчерпания многообразия, т.е. такие исчерпания, которые являются геодезическими в некоторой конформной исходной метрике. (По поводу возможности обобщений см. замечание 2 в конце работы.)

Будем говорить, что исчерпание $E$ имеет конформно-параболический тип (соответственно конформно-гиперболический тип), если существует полная, конформная исходной, метрика $g$, в которой исчерпание $E$ является геодезическим и интеграл (1) расходится (соответственно сходится).

Поскольку интеграл (1), как легко видеть, инвариантен относительно сферически конформньх замен метрики $g$, а любая метрика сферически конформно эквивалентна некоторой полной метрике, то имеет место

УТВЕРЖДЕНИЕ 3. Произвольное конформно-геодезическое исчерпание риманова многообразия имеет либо конформно-параболический, либо конформно-гиперболический mun.

Тогда теорема 1 может быть переформулирована следующим образом.

Теорема 2. Риманово многообразие $M$ является конформно-параболическим ( соответственно конформно-гиперболическим) тогда и только тогда, когда существует (соответственно любое) конформно-геодезическое исчерпание $M$ конформно-параболического типа (соответственно конформно-гиперболического ти$n a)$.

Отметим, что на конформно-параболическом многообразии существуют исчерпания как конформно-параболического, так и конформно-гиперболического типов (см. [5]).

В связи с утверждением 3 естественно возникает задача выявления геометрических характеристик (инвариантов), определяющих конформньй тип исчерпания.

Введем следующий конформньй инвариант произвольного исчерпания.

Назовем конформной изопериметрической размерностью исчерпания $E=\{D(t) \mid$ $\left.t \in \mathbb{R}_{+}\right\}$точную верхнюю грань таких чисел $p \geqslant 0$, для каждого из которых найдется конформная исходной метрика, в которой вьполняется неравенство вида (4) для областей $D=D(t)$ при всех достаточно больших значениях $t$.

На самом деле, для любого исчерпания конформная изопериметрическая размерность реализуется как изопериметрическая размерность исчерпания в некоторой полной, конформной исходной, метрике. Это, в частности, указьвается в следующих теоремах, дающих критерии конформного типа исчерпания. 
ТЕОРема 3. Для произвольного конформно-геодезического исчерпания $E$-мерного риманова многообразия $M$ следующие утверэдения әквивалентны:

1) Е имеет конформно-параболический тип;

2) конформная изопериметрическая размерность Е равна $n$;

3) существует полная, конформная исходной, метрика на $M$, в которой максимальная изопериметрическая функиия $P_{\max }$ исчерпания $E$ имеет вид $P_{\max }(x)=x^{(n-1) / n}\left(\right.$ как в $\left.\mathbb{R}^{n}\right) ;$ в частности, в этой метрике изопериметрическая размерность исчерпания равна $n$.

ТЕОремА 4. Для произвольного конформно-геодезического исчерпания Е риманова многообразия $M$ следуюшие утверэсдения әквивалентны:

1) Е имеет конформно-гиперболический тип;

2) конформная изопериметрическая размерность Е равна $+\infty$;

3) существует полная, конформная исходной, метрика на $M$, в которой максимальная изопериметрическая функиия $P_{\max }$ исчерпания $E$ имеет вид $P_{\max }(x)=x\left(\kappa а \kappa ~ в ~ H^{n}\right) ;$ в частности, в этой метрике изопериметрическая размерность исчерпания равна $+\infty$.

Доказательства теорем похожи, поэтому докажем, например, теорему 3. Импликация 1) $\Longrightarrow 3$ ) справедлива в силу леммы 2 , которую надо применить к функции $\widetilde{P}(x)$ вида $x^{(n-1) / n}$.

С другой стороны, если для $E$ имеет место утверждение 3$)$, то в некоторой полной, конформной исходной, метрике $g$ функция объема $V(r)$ геодезического шара радиуса $r$ при больших $r$ удовлетворяет дифференциальному уравнению $V^{(n-1) / n}(r)=V^{\prime}(r)$, решая которое при больших $r$ находим $V(r)=c \cdot r^{n}$ и $S(r)=n c \cdot r^{n-1}$. Следовательно, исчерпание $E$ конформно-параболично, т.е. 3$) \Longrightarrow 1$ ).

Далее, если $E$ обладает свойством 1 ), то конформная изопериметрическая размерность исчерпания $E$ согласно следствию 2 не больше $n$. Если же $E$ удовлетворяет 3 ), то его конформная изопериметрическая размерность, очевидно, не меньше $n$. Тем самым доказана эквивалентность всех утверждений 1)-3) теоремы 3.

С учетом теоремы 2 из теорем 3 и 4 соответственно получаем следующие критерии конформного типа риманова многообразия.

СлЕДСТВИЕ 3. Риманово многообразие размерности $n$ конформно-параболично тогда и только тогда, когда существует конформная исходной полная метрика, в которой его геодезическое исчерпание имеет конформную изопериметрическую размерность, равную $n$.

СлЕДСТВИЕ 4. Риманово многообразие конформно-гиперболично тогда и только тогда,когда в любой полной, конформной исходной, метрике его геодезическое исчерпание имеет конформную изопериметрическую размерность, равную $+\infty$.

ЗАмечАниЕ 2. Результаты данной работы, полученные для конформно-геодезических исчерпаний, можно распространить (без принципиальных усложнений в их доказательствах) на достаточно общие исчерпания. При этом, вместо функции $S(r)$ в метрике $g$ следует рассматривать ее аналог - функцию

$$
S_{h}(r)=\int_{h=r}|\nabla h|^{n-1} d \sigma,
$$


где $h$ - достаточно регулярная функция, порождающая данное исчерпание (как семейство множеств $D(r)$, на которых $h<r),|\nabla h|$ - модуль градиента функции $h, d \sigma$ - элемент $(n-1)$-меры в метрике $g$ соответственно.

Авторы благодарят Н. А. Зорич за помощь в подготовке электронной версии текста этой заметки.

\section{СПИСОК ЦИТИРОВАННОЙ ЛИТЕРАТУРЫ}

[1] Ahlfors L. Zur Theorie des Überlagerungsflächen // Acta Math. 1935. V. 65. P. 157-194.

[2] Gromov M. Structures métriques pour les variétés riemanniennes. Notes du cours rédigé par J. Lafontaine et P. Pansu. Paris: CEDIC/Fernand-Nathan et Soc. Math. de France, 1981.

[3] Pansu P. Quasiconformal mappings and manifolds of negative curvature // Lecture Notes in Math. 1986. V. 1201. P. 212-229.

[4] Зорич В. А., Кесельман В. М. О конформном типе риманова многообразия // Функцион. анализ и его прилож. 1996. Т. 30. № 2. С. 40-55.

[5] Grimaldi R., Pansu P. Sur la croissance du volume dans une classe conforme // J. Math. Pures Appl. (9). 1992. V. 71. № 1. P. 1-19.

[6] Ahlfors L. Sur le type d'une surface de Riemann // C. R. Acad. Sci. Paris. Sér. A. 1935. V. 201. P. $30-32$.

Московский государственный университет им. М.В. Ломоносова

Поступило

Кемеровский государственный университет 24.06.96 